This item was submitted to Loughborough's Research Repository by the author.

Items in Figshare are protected by copyright, with all rights reserved, unless otherwise indicated.

\title{
A new method to evaluate a trained artificial neural network
}

PLEASE CITE THE PUBLISHED VERSION

PUBLISHER

(C) IEEE

VERSION

VoR (Version of Record)

LICENCE

CC BY-NC-ND 4.0

\section{REPOSITORY RECORD}

Yang, Yingjie, Chris J. Hinde, and David Gillingwater. 2019. "A New Method to Evaluate a Trained Artificial Neural Network". figshare. https://hdl.handle.net/2134/4122. 
This item was submitted to Loughborough's Institutional Repository (https://dspace.lboro.ac.uk/) by the author and is made available under the following Creative Commons Licence conditions.

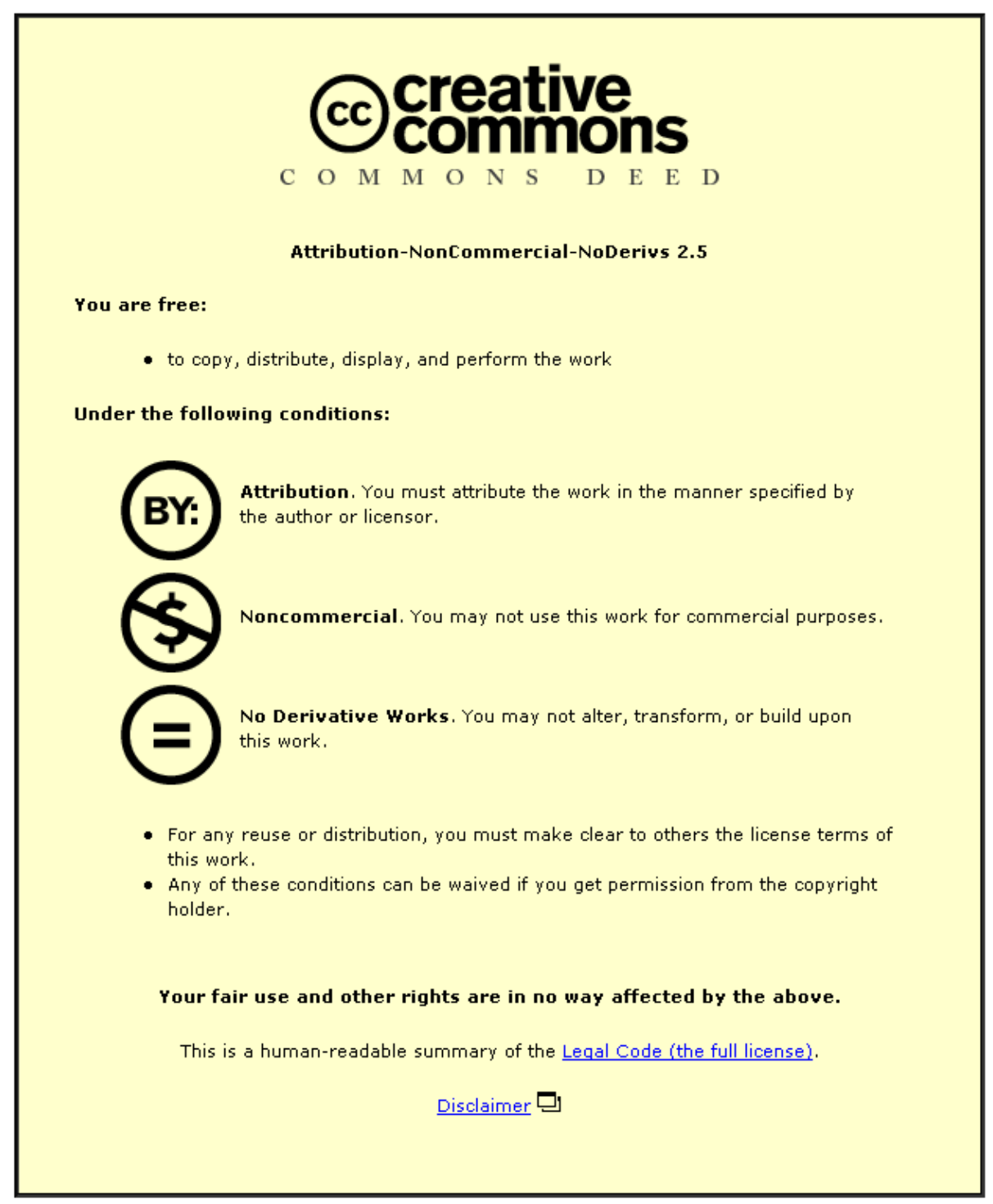

For the full text of this licence, please go to: http://creativecommons.org/licenses/by-nc-nd/2.5/ 


\section{A New Method to Evaluate A Trained Artificial Neural Network}

\author{
Yingjie Yang \\ Loughborough University \\ Department of Civil and \\ Building Engineering \\ Loughborough \\ Leics. \\ LE11 $3 T U$ \\ $U K$ \\ Y.Yang@lboro.ac.uk
}

\author{
Chris Hinde \\ Loughborough University \\ Department of Computer \\ Science \\ Loughborough \\ Leics. \\ $L E 113 T U$ \\ $U K$ \\ C.J.Hinde@lboro.ac.uk
}

\author{
David Gillingwater \\ Loughborough University \\ Department of Civil and \\ Building Engineering \\ Loughborough \\ Leics. \\ LE11 $3 T U$ \\ $U K$ \\ D.Gillingwater@lboro.ac.uk
}

\begin{abstract}
In comparison with traditional local sample testing methods, this paper proposes a new approach to evaluate a trained neural network. A new parameter is defined to identify the different potential roles of the individual input factors based on the trained connections of the nodes in the network. Compared with field-specific knowledge, the dominance of individual input factors can be checked and then false mappings satisfying only the specific data set may be avoided.
\end{abstract}

\section{Introduction}

One of the problems in the application of an artificial neural network (ANN) in engineering practice is the difficulty in verifying its function after the training stage. The usual way is to keep some sample data out of its training set so as to test it later. However, it is difficult to decide how much data should be left out since this would reduce the limited data in the training set. On the other hand, it is impossible to test every possible situation in that the artificial neural network is applied usually when we do not know all possible situations. Hence, a simple method which links the general field knowledge and the network structure is better for evaluating the operation of an artificial neural network. Here, a new approach based on the analysis of network structure and field knowledge is presented to help test the function of the artificial neural network.

In the case that the outputs of the Neural Network are essentially Boolean, Fletcher and Hinde [1] show that the network hypothesis can be expressed as a set of rules. In another piece of work, still focusing on networks with Boolean outputs Fletcher and Hinde [2] showed that although a rule based interpretation was useful in many cases, pattern based systems produced an inordinate number of rules. They introduced a system to invert the network efficiently and reliably. The two systems were complementary in that inverting a rule based system tended to produce little useful information, and conversely converting a pattern based system into rules also resulted in a confused situation. The proposed system gives a different view of the network and furthermore gives information about networks with Real outputs and Real inputs.

In comparison with the current local sample testing approach, this paper proposes a new global method to validate the trained artificial neural network. With the analysis of the BP network structure, this paper puts forward a new concept - Potential Relative Strength of Effect (PRSE) and the Global PRSE. They provide a link between network structure and field knowledge, which serves as an audit of the trained neural network.

\section{Traditional Validation}

The capability to learn from examples by machine without prerequisite knowledge about the specific problem has enabled ANN to become a popular model in engineering applications. Many engineering problems, such as civil engineering, environmental engineering and transportation engineering, involve a number of uncertain mechanisms which complicate the interactions between their different factors. These unknown mechanisms bring the "black box" problems suitable for an ANN to interpret. Because of this kind of incomplete knowledge with respect to the domain problems, the significance of the validation of a trained network appears more important than ever.

There have been numerous different ANN models and a variety of methods for training them; however, the validation of a trained neural network is still carried out using mainly the local sample testing method. This approach randomly separates the entire available data set 
into two different sets: the training set and the test set. The training set is then used to train the network and the test set is adopted to test the function of the trained network, as shown in Figure 1.

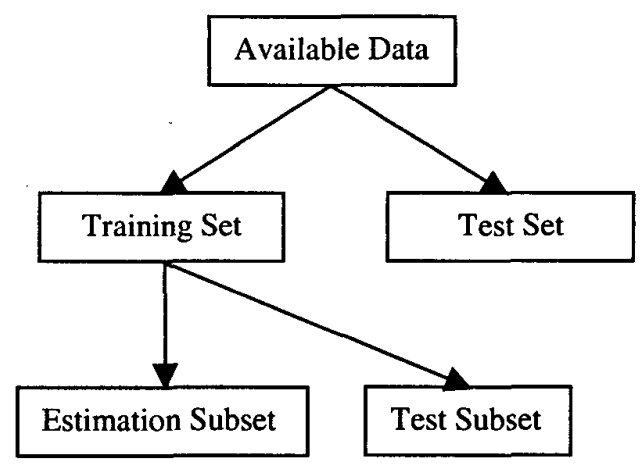

Figure 1: The partition of the available data

In addition to the two sets partition, there is some other validation method which divides the data further, such as the so called cross-validation [3]. This testing method comes from the standard statistics tool cross-validation [4]. After the available data set is randomly partitioned into a training set and a test set, the training set is divided further into two disjoint subsets: an estimation subset and a validation subset. The estimation subset is used to select the model (structure and parameters of the network), but the validation subset is used to test or validate the model. The external test set serves for checking the generality of the trained network.

It is obvious that this kind of validation needs a large amount of data. For the available data set, it has to be divided into two or three separate parts and the real data for training the network is only one part of them. However, the data requirement for training a neural network efficiently is also very high. According to the Vapnik-Chervonenkis (VC) dimension [5], the following rule applies [6]:

Let $N$ denote a multi-layer feed forward network whose neurons use a sigmoid activation function

$$
\varphi(v)=\frac{1}{1+e^{-v}}
$$

The VC dimension of $N$ is $O\left(W^{2}\right)$, where $W$ is the total number of free parameters in the network.

The number of samples needed to learn a mapping reliably is proportional to the VC dimension of that mapping [3], hence the required number of samples for training a neural network is also $O\left(W^{2}\right)$.
In engineering practice, it is sometimes difficult to find sufficient data to train networks. Most data comes from costly measurements carried out on site and involve various uncertainties and complex interactions. With the limited data set, it is difficult to know what the effect would be if only a small part of the available data is used to train the network. There would be two kinds of data set available: ideally distributive data without redundancy and data with repeat and redundancy. For the first group, the pattern existing in the testing data would not be able to be represented in the network trained with the other partial data; and the good agreement for the redundant data in the second group is not reliable for data not considered. The crucial problem for current validation methods is its basic assumption:

1. The data in the testing set is representative enough for covering the interesting scope.

2. All the patterns in the testing set have been represented in the training set.

In fact, the reason for ANN to be applied is precisely because there is no clear understanding about the mechanism reflected by the data set. Therefore, it is difficult to know if the testing data set has included all possible situations. Considering the potential size of neural networks applied in engineering practice, it is impossible sometimes for the testing set to include all the possible situations within the scope of interest. With limited available data, the more the testing samples are, the less the training data would be and then the poor reliability of the trained network.

Therefore, a better way to check and validate the trained neural network should be to make full use of the available data at the training stage and find the false mapping without or with less involvement of the mass validation data. Here, a new parameter is put forward to find a different approach to evaluate a trained neural network.

\section{PRSE and GPRSE}

The Kolmogorov mapping neural network existence theorem [7] has proved that: given any continuous function

$$
\begin{aligned}
& \varphi: I^{n} \rightarrow R^{m} \\
& Y=\varphi(X)
\end{aligned}
$$

$\varphi$ can be implemented exactly by a three-layer neural network having $n$ input nodes, $2 n+1$ hidden nodes and $\mathrm{m}$ output nodes. Thus a three layer neural network has the capability to implement any continuous mapping. It is well known that the knowledge representation of ANN is in the form of the connection weights between the nodes of different layers. Hence the relative significance of the 
individual input nodes for the output value could be identified from the distribution of these connection weights. Thus a 'Relative Strength of Effect' (RSE) can be computed. It provides the measure of the contribution which every parameter makes to the system $[8,9]$.

If a reference data set is available, given by $S=\left\{s_{1}, s_{2}, s_{3}\right.$, $\left.\ldots, \mathbf{s}_{\mathrm{j}}, \ldots \mathbf{s}_{\mathbf{r}}\right\}$, where $\mathrm{s}_{\mathrm{j}}=\{\mathrm{X}, \mathrm{Y}\}, \mathrm{X}=\left\{x_{1}, x_{2}, x_{3}, \ldots, x_{p}\right\}, \mathrm{Y}=\left\{y_{1}\right.$, $\left.y_{2}, y_{3}, \ldots, y_{q}\right\}$, then a neural network trained using a BP algorithm will yield the $\mathrm{RSE}_{\mathrm{ki}}$ for input $\mathrm{i}$ on output $\mathrm{k}$ as:

$$
\begin{aligned}
& \operatorname{RSE}_{\mathrm{kj}}=C \sum_{\mathrm{jn}} \sum_{\mathrm{jn}-1} \ldots \sum_{\mathrm{j} 1} W_{j_{n} k} \mathrm{G}\left(e_{k}\right) W_{j_{n-1} j_{n}} \mathrm{G}\left(e_{j_{n}}\right)
\end{aligned}
$$

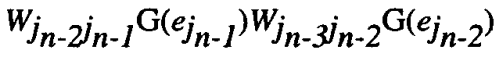

$$
\begin{aligned}
& \ldots W_{i j_{1}} \mathrm{G}\left(e_{j_{1}}\right)
\end{aligned}
$$

where $C$ is the normalised constant controlling the maximum absolute value. The function $G$ denotes the differentiation of activation functions. $W$ is a connected weight and $e$ is the input value at its corresponding node.

The larger the absolute value, the greater the effect which the corresponding input factor has on the output unit. The sign indicates the direction of influence; thus a positive action applies to the output when RSE $>0$, and vice verse. RSE is a dynamic parameter changing with the variance of the input values of the network. RSE is sensitive to a change in sign of the connecting weight and output of each individual node. However, the absolute value of the connecting weight and individual node is more meaningful in the wider context, indicating the potential maximum capability for a relevant factor to control the corresponding output.

Hence a new parameter can be defined which can judge the degree of importance of a variable on the system, measuring the relative significance of inputs with respect to outputs in the trained neural network. On the basis of RSE, the Potential RSE and Global Potential RSE (GPRSE) have been defined.

Definition: For a neural network trained using the BP algorithm and for a given reference data set $S=\left\{\mathbf{s}_{1}, \mathbf{s}_{2}, \mathbf{s}_{\mathbf{3}}\right.$, $\left.\ldots, \mathbf{S}_{\mathbf{j}}, \ldots \mathbf{s}_{\mathbf{r}}\right\}$, where, $\mathbf{s j}=\{\mathbf{X}, \mathbf{Y}\}, \mathbf{X}=\left\{x_{1}, x_{2}, x_{3}, \ldots, x_{p}\right\}$, $\mathbf{Y}=\left\{y_{1}, y_{2}, y_{3}, \ldots, y_{q}\right\}$ :

$$
P R S E_{k i}=\frac{\sum_{j_{n}} \sum_{j_{n-1}} \cdots \sum_{j_{1}}\left|W_{j_{n} k}\right| \cdot\left|G\left(e_{k}\right)\right| \cdots\left|W_{i j_{1}}\right| \cdot\left|G\left(e_{j_{1}}\right)\right|}{\sum_{i} \sum_{j_{n}} \sum_{j_{n-1}} \cdots \sum_{j_{1}}\left|W_{j_{n} k}\right| \cdot\left|G\left(e_{k}\right)\right| \cdots\left|W_{i j_{1}}\right| \cdot\left|G\left(e_{j_{1}}\right)\right|}
$$

$$
\operatorname{GPRSE}_{k i}=\frac{\sum_{j_{n}} \sum_{j_{n-1}} \cdots \sum_{j_{1}}\left|W_{j_{n} k}\right| \cdot\left|W_{j_{n-1} j_{n}}\right| \cdots\left|W_{i j_{1}}\right|}{\sum_{i} \sum_{j_{n}} \sum_{j_{n-1}} \cdots \sum_{j_{1}}\left|W_{j_{n} k}\right| \cdot\left|W_{j_{n-1} j_{n}}\right| \cdots\left|W_{i j_{1}}\right|}
$$

where, the function $G$ denotes differentiation of the activation function. $W$ is a connected weight and $e$ is the input value in its corresponding node.

PRSE and GPRSE are measures of the absolute value of every weight and node value. The absolute influence of every connection and node is thus accumulated. Hence, no matter which factors are dominant, the contribution of every factor will be incorporated within the calculation of PRSE and GPRSE.

Compared with RSE, the removal of the different signs makes the PRSE and GPRSE less sensitive to a small change of input, thus they are measures of the potential within a wider scope of neighbourhood rather than a detailed trend at a specific point.

\section{Validation with PRSE}

In engineering practice, the exact independence between different factors represented by RSE is difficult to know because of our ignorance of the complicated interactions. However, the statistics and expertise often have the ability to know roughly which factor is dominant and their relative importance index. In this sense, it is possible to know the GPRSE for the interest scope even before we begin to train the neural networks. This provides us with an alternative for evaluating and validating a trained neural network.

As we know, a suitably trained neural network is able to map the relationships between its input factors and output attributes. This efficient mapping has a precondition: the network can recognise the different roles of the different factors for its mapping function. An important factor should be able to play a significant role when a suitable input is fed into the network. The GPRSE should agree with the field knowledge obtained from statistics or expertise. Hence, a comparison between the GPRSE and the field knowledge about the dominance of different factors in the system would help us to evaluate the trained network.

The GPRSE is defined as a global parameter within the scope of interest, and is capable to indicate the general significance of the individual factors. However, some relationships may be very complicated and a general validation is not sufficient to audit network behaviour, and the PRSE for some special points would be helpful to validate its function in some special segments. As we know from Equation (4), the PRSE relies on the specific 
point in input space but is not so sensitive to its position changes like RSE. It reflects a potential dominance within a wider scope of the neighbourhood of the input point. The scheme to evaluate and validate a trained neural network with GPRSE and PRSE is shown in Figure 2.

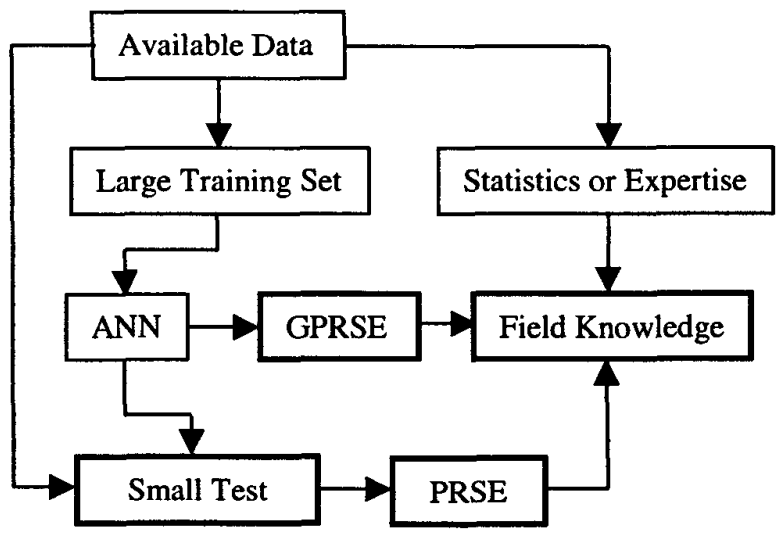

Figure 2: The evaluation scheme

Similar to the traditional test, the available data are separated into two different sets: the training set and the test set. However, the number of the samples in the test set could be reduced dramatically if suitable field knowledge about the specific problem is possible. Although satisfying the training data, the false mapping would produce some false details in the resultant mapping relationships. There would be some distortions of the connection weights to provide such kinds of false detail. These changed connections would change their absolute significance and thus be reflected in the changes of GPRSE. Therefore, the disagreement between the GPRSE from the trained network and the field knowledge from statistics and expertise indicates the unreliable mapping of the trained network.

The trends audit with GPRSE would prevent most of the false mappings. Only the false mapping that possesses similar GPRSE values with our knowledge could survive this evaluation. The PRSE audit could be conducted with some key samples where field knowledge is possible. Compared with RSE, PRSE reflects the potential dominance of the factor within a wider scope and hence provides an indicator about local distortions. Those false mappings satisfying the GPRSE would be exposed if the specific field knowledge is available in a few local segments. In this way, the possible false mappings could be filtered dramatically without using large amounts of testing data. Hence, most data could be adopted as training data and the feasibility of the trained network could be improved dramatically. It should be noted that the small test data set for PRSE needs only the relevant dominance rather than the corresponding output. However, it can also serve for traditional testing as a supplement to the proposed method if their outputs are known.

In this way, the available data set is fully applied to the training stage and hence improves the reliability of the trained network considerably. The evaluation and validation of the trained network is carried out mainly with the global parameters GPRSE, as well as the PRSE for some key points.

\section{Application Example}

To illustrate the applicability of the proposed evaluation method, a simple example for partition is illustrated in Figure 3. The points in Figure 3 belong to two different parts. The input factors of the 8 samples are the vertices of the two parts, and the output is 0 for left and 1 for right part. Obviously, the maximum significance of the two dimensions $(x, y)$ are exactly the same: no influence to the output in the left side of $\mathrm{AB}$ and the same importance along the boundary interface. Therefore, the field knowledge advises:

$$
\begin{aligned}
& \text { GPRSE }_{\mathrm{x}}=\text { GPRSE }_{\mathrm{y}} \\
& \operatorname{PRSE}_{\mathrm{x}}^{\mathrm{a}}=\operatorname{PRSE}_{\mathrm{y}}^{\mathrm{a}}
\end{aligned}
$$

where, 'a' represents an arbitrary point in the two parts of Figure 3.

For a simple problem like this, it is applicable to test the trained neural network in the whole scope of interest. Therefore, the traditional testing method is applied here to validate the applicability of the new apporach proposed in this paper.

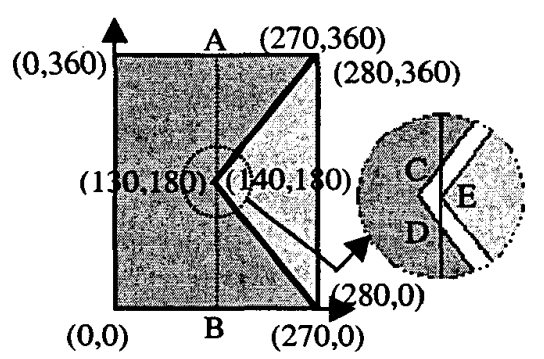

Figure 3: The partition problem

As aforementioned, a three layer network (one hidden layer) has the ability to approach any continuous mapping. Hence, we investigate only the structure with one hidden layer here. The structure with $3,4,5,6,7,8,9,10$ and 15 hidden nodes are studied with a random intialised connection weights within $[0,1]$. For every structure, 10 converged networks with different initial connection weights are established. 
Coincidentally, the acceptable and false mappings are equivalent in the 90 established networks: 45 for true and the other $\mathbf{4 5}$ for false. The true mapping appears similar to each other, as shown in Figure 4. However, the false
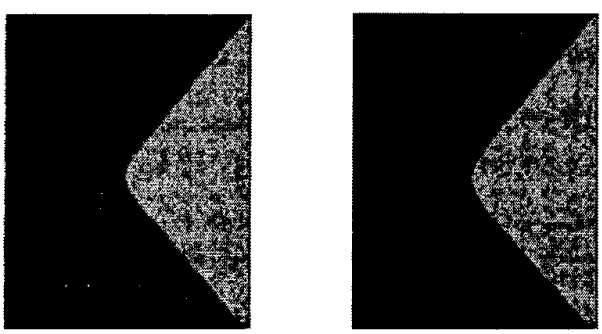

Figure 4: The results of two acceptable mappings
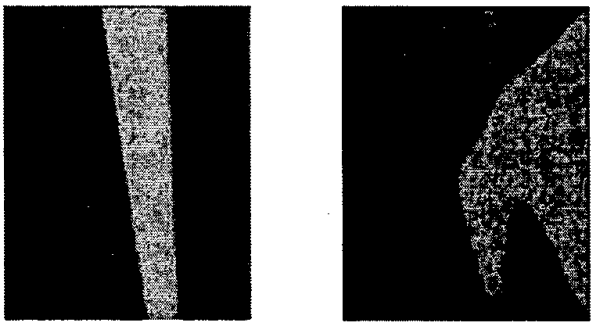

Figure 5: Some results of the false mappings

mapping shows the diversity of the possible false solutions; two examples are shown in Figure 5. It should be noted that all the false mappings in this experiment converge well and have the potential to continue convergence further.

Obviously, the results of the acceptable mapping and the false maping are very different. This conclusion could be found easily for a simple problem by this exhaustive testing method. However, it is not so easy for a complex engineering problem where a large scale network is involved. Because of the potential complexity of engineering problems, it is not possible to test every possible situation. The only way to test it with the traditional method is to keep a large amount of sample data out of the training set and then test the trained network with this reserved data set. As aforementioned, this operation reduces the available data set for training the network and cannot prove the applicability of the network for the data not included in the testing set. For example, the false mappings in Figure 5 may find a number of satisfactory test results if the test points are not located in the distorted areas. Hence, the conclusions based on this kind of local sample testing are not reliable if we cannot make an efficient distribution of the test data.

Based on the trained neural networks, the GPRSE results for the acceptable mapping and false mapping are calculated and illustrated in Figure 6.
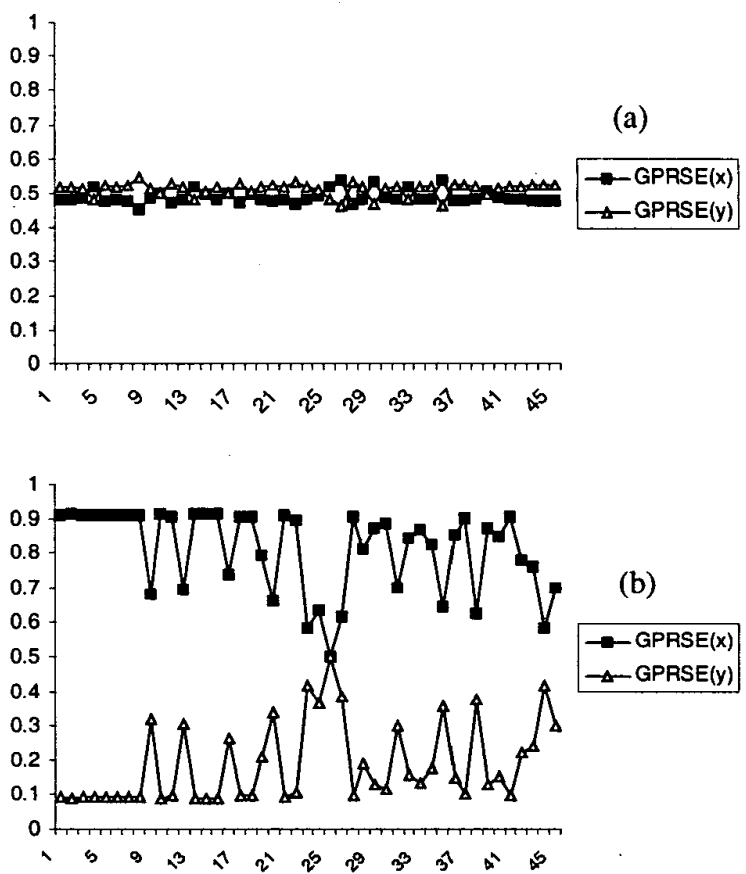

Figure 6: The comparison of GPRSE

Figure 6(a) shows the GPRSE for the acceptable group, and Figure $6(\mathrm{~b})$ is the GPRSE for the false mapping group. The GPRSE pairs for $x$ and $y$ distributed along the line of GPRSE $=0.5$ proves that the GPRSE ${ }_{\mathrm{x}} \cong$ GPRSE for the acceptable group. On the contrary, most GPRSE pairs for the false mapping group are far from each other in Figure 6(b). Obviously, the false mapping is reflected well by their GPRSE, that is: GPRSE ${ }_{\mathrm{x}} \neq$ GPRSE $_{\mathrm{y}}$ for the false mapping. Compared with Equation (6), it is reasonable to evaluate the trained network by means of GPRSE: the network would result in false mapping if its GPRSE pairs for $\mathrm{x}$ and $\mathrm{y}$ have a large distance from each other. Among the 90 trained networks, only one is an exception to this rule, and the probability for this rule to identify the false mapping is larger than 0.99 .

The exception of the pure GPRSE identification is in the false mapping group. As demontrated in Figure 6(b), network 26 ( 6 hidden nodes) possesses a GPRSE pair of 0.499 for $x$ and 0.501 for $y$. According to the first equation in Equation (6), this network should be an acceptable one. 
However, its exhaustive testing shows a distorted result as shown in Figure 7.

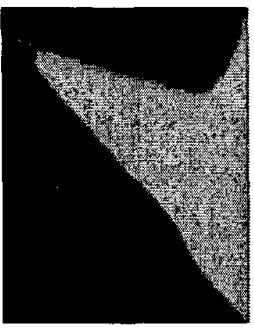

Figure 7: The result of the exception case

Obviously, this mapping forms another kind of pattern with equivalent GPRSE pairs for this problem. Hence the GPRSE identification does not work in this special case. However, although this pattern keeps the equivalent GPRSE pairs, its local distortion could be found with some local PRSE evaluation. For example, we select one centre point in every partition to undertake the PRSE analysis. Here the points are $(70,180)$ for the left partition and $(210,180)$ for the right part. The result of their mapping from the exceptional network 26 in the false mapping group and a normal network 11 in the acceptable group are listed in Table 1. The column 'Oi' is the ideal output, and 'On' is the real output from the trained network.

Table 1: The comparison between the two cases

\begin{tabular}{lrrrrrrrr}
\hline Group & No. $x$ & \multicolumn{1}{c}{$y$} & Oi & On & PRSE $_{x}$ & PRSE $_{y}$ \\
\hline FALSE & 26 & 70 & 180 & 0 & 0 & 0.43 & 0.57 \\
FALSE & 26 & 210 & 180 & 1 & 1 & 0.71 & 0.29 \\
Accept & 11 & 70 & 180 & 0 & 0 & 0.48 & 0.52 \\
Accept & 11 & 210 & 180 & 1 & 1 & 0.48 & 0.52 \\
\hline
\end{tabular}

The two points selected here located in the centre of the two parts and the variance of $x$ or $y$ would not causes the change of the output within their neighborhood. Therefore, their PRSE values should be equivalent to each other. Obviously, network 11 in the acceptable group agrees with this analysis well, but the exceptional case in the false mapping group does not meet this requirement. However, the mapping results of both cases are satisfactory. Obviously, the traditional testing method fails to find the problem in this case, and the proposed PRSE analysis works well.

Generally, the GPRSE identification works well if the general trends are sufficiently clear. For more complicated problems, the general trends may not be so clear and then PRSE may be involved to validate it further.

\section{Conclusions}

An exhaustive testing of possible situations for a neural network by means of testing samples is neither possible nor necessary. It would reduce the limited available data for training the neural network in engineering practice. Hence this paper presents a new methodology to combine the field knowledge on the trends analysis with the network validation process.

As a global parameter, the GPRSE is a very good indicator for the behaviour of a trained neural network. As the index of the importance of the input parameters on determining the output, GPRSE should agree with the special field knowledge of the relative role of the individual input parameter. For a more complicated problem where GPRSE is not sufficiently clear, the PRSE analysis serves for further investigation. Therefore, an analysis of the specific field knowledge combined with a few sample tests would give a better audit to the efficiency of the trained artificial neural network. In this way, the available data could be fully applied to the training stage and the validation is simple and efficient.

[1] Fletcher, G P and Hinde, C.J., 'Using Neural Networks as a Tool for Constructing Rule Based Systems", Knowledge Based Systems, Vol 8, No.4, 1995, pp 183-189.

[2] Fletcher, G.P. and Hinde, C.J., 'Producing Evidence for the Hypotheses of Large Neural Networks", Neurocomputing , Vol 10, 1996, pp 359-373.

[3] S. Haykin Neural networks: a comprehensive foundation, Prentice-Hall, New Jersey, 1999.

[4] M. Stone, "Cross-validatory choice and assessment of statistical predictions", Journal of the Royal Statistical Society, vol. B36, 1974, pp. 111-133.

[5] V.N. Vapnik and A. Y. Chervonenkis, "On the uniform convergence of relative frequencies of events to their probabilities", Theoretical Probability and lts Applications, vol.17, 1971, pp.264-280.

[6] P. Koiren and E.D. Sontag, "Neural networks with quadratic VC dimension", Advances in Neural Information Processing Systems, Cambridge, MA:MIT Press, vol..8, 1996, pp.197203.

[7] R. Hecht-Nielsen, "Kolmogorov's mapping neural network existence theorem", Proc. IEEE First Int. Conf. On Neural Networks, San Diego, Vol. III, 1987, pp.11-14.

[8] Y. Yang and Q. Zhang, "A hierarchical analysis for rock engineering using artificial neural network", Rock Mechanics \& Rock Engineering. Vol.30, No.4, 1997, pp.207-222.

[9] Y. Yang and Q. Zhang, "A new method for the application of artificial neural networks to rock engineering systems", International Journal of Rock Mechanics and Mining Science. Vol.35, No.6, 1998, pp.727-745. 